\title{
Picosecond laser ablation system with process control by Optical Coherence Tomography
}

\author{
Piotr Targowski ${ }^{* a}$, Roman Ostrowski ${ }^{b}$, Jan Marczak ${ }^{b}$, Marcin Sylwestrzak ${ }^{\mathrm{a}}$, Ewa A. Kwiatkowska ${ }^{\mathrm{a}}$ \\ ${ }^{a}$ Institute of Physics, Nicolaus Copernicus University, ul. Grudziadzka 5, PL-87 100 Toruń, Poland \\ ${ }^{\mathrm{b}}$ Institute of Optoelectronics, Military University of Technology, ul. S. Kaliskiego 2, PL-00 908 \\ Warszawa, Poland
}

\begin{abstract}
A work station for controlled ablation of varnish from pictures is described. An apparatus utilises forth harmonic of picosecond Nd:YAG laser, computer-controlled sliding object stand and OCT inspection system. An influence of laser parameters on ablation process is discussed. Method and results of space-resolved ablation rate determination are shown.
\end{abstract}

Keywords: laser cleaning, ablation, Optical Coherence Tomography (OCT), artwork, easel painting

\section{INTRODUCTION}

In this contribution we describe an apparatus for precise laser ablation of delicate layers, like varnish on pictures. This specific case is very demanding. First of all any changes in colour of remaining varnish layer as well as underneath paint layers are unacceptable. This effect may be induced photochemically or thermically. In the first case strong absorption of the radiation used will eliminate its influence on underlying strata. The thermal effect is limited to so called heat affected zone (HAZ). In addition to colour change, a mechanical damage caused by overheating of the structure adjacent to ablated region should be considered also. All kinds of treads must be carefully eliminated in order to make laser ablation of varnish commonly accepted alternative to chemical and/or mechanical treatments [1].

Since the varnish ablation process is obviously irreversible its effective monitoring is very important to make it safe and trusted. As we showed previously [2-6] optical coherence tomography (OCT) originated from medicine diagnostic method for examination and imaging of cross-sections of weakly absorbing objects can be used for this task. OCT utilises infrared light for non-invasive structure examination and has been under consideration for the examining of objects of art since 2004 [7-10]. In this case the in-depth (axial) resolution is obtained by means of interference of light of high spatial (to ensure sensitivity) and very low temporal coherence (to ensure high axial resolution). In practice, IR sources of bandwidths from 25 to $150 \mathrm{~nm}$ are utilised. Resolutions obtained range from 15 down to $2 \mu \mathrm{m}$ in the media of refracting index equal 1.5 .

In this contribution we expand application of OCT to space resolved determination of ablation rates, separately for every point of examined area. Such data help in better understanding of the ablation process, fine tuning the laser and finally permit increase of the safety of the ablation process.

\section{METHODOLOGY}

\subsection{Laser ablation}

Laser ablation term generally refers to the removal of a material (top layer) during photo-thermal or photo-chemical interaction of laser radiation with matter.

In photo-thermal processes an absorbed energy of laser radiation is converted into heat. Due to strong absorption of laser radiation in surface layer, a high and sudden increase of temperature occurs which in turn causes material evaporation or thermal stresses resulting in spallation. If energy of laser radiation incident on material's surface is high enough, the temperature of the surface exceeds boiling point which results in rapid evaporation of the material. The above processes are generally referred to as photo-thermal ablation. This kind of ablation mechanism dominates during

*ptarg@fizyka.umk.pl; phone +48 56611 3206; fax +48 566225397

P. Targowski et al. "Picosecond laser ablation system with process control by Optical Coherence Tomography", Proc of SPIE 7391, p. $7391-15$

Copyright 2009 Society of Photo-Optical Instrumentation Engineers. One print or electronic copy may be made for personal use only. Systematic reproduction and distribution, duplication of any material in this paper for a fee or for commercial purposes, or modification of the content of the paper are prohibited. 
removing materials like metals or some polymers. Another process, a photo-chemical ablation consists in breaking off bonds in molecular chains of removed material by incident photons. To do so, photon energy must be higher than bonding energy. In effect, removing of material occurs as a result of fragmentation of molecules without significant thermal damage.

Among parameters describing ablation process the most important seem to be absorption coefficient $\alpha$ and heat diffusivity $\kappa$. High absorption coefficient and low heat diffusivity result in high efficiency of laser ablation process. Short laser pulses (pulse duration is shorter than thermal relaxation time of ablated material) are conducive to material ablation due to energy confinement in a thin layer of the material. For longer laser pulses (pulse durations higher than thermal relaxation time), due to heat transport, the absorbed energy will be dissipated to the vicinity of the interaction zone. To facilitate the photo-ablation process with minimal thermal effects, laser pulse duration must therefore be shorter than thermal relaxation time of the material.

Therefore, high efficiency of laser ablation of materials requires a laser to be operated at the shortest available wavelengths and with pulse of nanosecond duration. In such a case time of interaction is so short that the energy of laser pulse is fully absorbed and ablation process is finished before heat diffusion occurs. In this conditions the head effected zone (HAZ) is minimal. Therefore high ablation efficiencies and acceptable processing precisions can be achieved without any thermal deterioration of surrounding material like melting, exfoliation, spallation or re-crystallization.

Since laser systems with pulse duration from femtosecond range are available on the market, these lasers are also worth consideration. Very attractive for varnish removal seems to be as well Er:YAG laser operated at $2.94 \mu \mathrm{m}$. This wavelength is in resonance with the vibrational energy of $\mathrm{OH}-$ and $\mathrm{NH}-$ bonds, which leads to very strong absorption of laser radiation, and thus to confinement of the laser energy and selective ablation of thin surface layers. However, experiments performed previously have shown $[5,11]$ that interaction of varnish layer with long laser pulses (of duration of several hundred microseconds) may results in melting down of removed material and further, in typical detrimental thermal effects, such as carbonization, discoloration and even burning. On the other hand, short interaction with high energy Q-switched laser pulses may results in ablation of material but with side effects caused by associated shock waves.

\subsection{Laser ablation system}

Diode-pumped picosecond Nd:YAG laser type of PL 2210 from Ekspla (Lithuania) has been used in experiment. Although this system is able to generate four harmonic wavelengths (1064, 532, 355 and $266 \mathrm{~nm}$ ), only the forth harmonic at $266 \mathrm{~nm}$ has been used to ensure minimal size of HAZ. This ultraviolet radiation is strongly absorbed by all varnishes under consideration and underneath layers are thus effectively isolated from any photochemical impact from the laser beam. The laser was running in passive mode locking regime: a sequence of laser pulses with energy of about $0.5 \mathrm{~mJ}$, duration slightly below $70 \mathrm{ps}$, and constant repetition rate of $1 \mathrm{kHz}$ was generated.

Laser beam was delivered to the surface to be ablated by a set of flat and totally reflecting mirrors and finally, through a focusing lens made of $\mathrm{CaF}_{2}$ and with a focal length of $40 \mathrm{~cm}$. The lens focused laser radiation on varnish sample placed on the computer controlled sliding $\mathrm{X}-\mathrm{Y}$ table. The same computer ensured synchronization with laser operation: by means of user interface a rectangular area of $30 \times 5 \mathrm{~mm}$ at sample surface was determined and then it was ablated by scanning with laser beam in fully automated manner. The table was translated in X and Y directions with fixed step of $20 \mu \mathrm{m}$ and fixed velocity of $2,500 \mathrm{steps} / \mathrm{sec}(50 \mathrm{~mm} / \mathrm{s})$. Due to the durability of these parameters and fixed pulse repetition rate changes in the laser spot size at sample resulted in different overlap factors. Thus the adjustment of laser fluency interacting with varnish was attained by an alteration of lens position in relation to the sample. Four lens locations were used in experiment, which resulted in laser spot size diameters on the sample of about 100, 130, 185 and $255 \mu \mathrm{m}$ and fluencies of $6.5,3.7,1.8$ and $1.0 \mathrm{~J} / \mathrm{cm}^{2}$, respectively. Therefore, for applied X-Y table translation parameters and these spot sizes the overlap factors were within the range of 50 to $80 \%$.

The general view of the whole ablation system, including laser, X-Y table, delivering mirrors and supporting structure, together with basic modules of OCT inspection system, are presented in Figure 1. The ablating system was designed and developed at Institute of Optoelectronics, Military University of Technology in Warsaw, Poland [12].

P. Targowski et al. "Picosecond laser ablation system with process control by Optical Coherence Tomography", Proc of SPIE 7391, p. $7391-15$

Copyright 2009 Society of Photo-Optical Instrumentation Engineers. One print or electronic copy may be made for personal use only. Systematic reproduction and distribution, duplication of any material in this paper for a fee or for commercial purposes, or modification of the content of the paper are prohibited. 


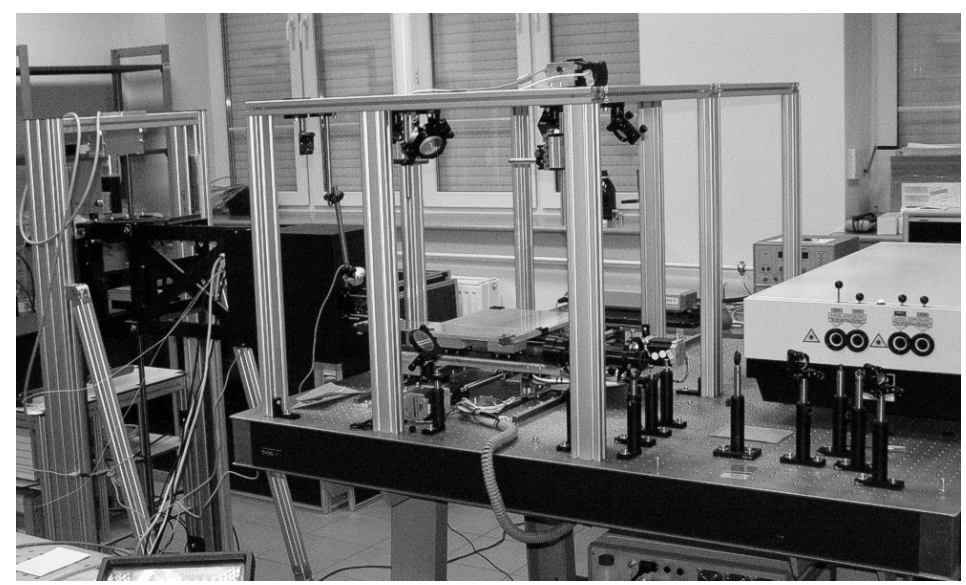

Fig. 1. The general view of the laser ablation system together with the OCT inspection system

\subsection{OCT inspection system}

To adopt proper ablation conditions the reliable process monitoring system is necessary. In the device described a prototype, high resolution Spectral OCT imaging system was employed (Fig. 2). The device was constructed at the Nicolaus Copernicus University in Torun (Poland). It is capable of creating cross-sectional images (tomograms) of objects weakly absorbing light (e.g. varnish layer) with axial resolution of $4 \mu \mathrm{m}$. It comprises a broadband infrared light source (LS, $\lambda_{c}=845 \mathrm{~nm}, \Delta \lambda=107 \mathrm{~nm}$ ) build of two superluminescent diodes coupled together. Then light of high spatial but low temporal coherence is launched into single mode optical fibre and passes through an optical isolator (OI), employed to protect the source from back-reflected light. The major part of the tomograph is in this set-up a fibre optic Michelson interferometer. It comprises a 50:50 fibre coupler (FC) dividing light into two arms: a reference one and an object one. The reference arm is built of a polarization controller (PC) which provides optimal conditions for interference, a neutral density filter (NDF) for adjustment of the power of light to achieve the shot-notice-limited detection, a block of glass (DC) acting as a dispersion compensator and the stationary reference mirror (RM). The object arm comprises a lens forming a narrow probing beam and transversal scanners (X-Y) responsible for scanning this beam across the sample. The light beams: back-reflected from the reference mirror and backscattered or/and reflected from the element of object's structure return to the coupler and interfere.

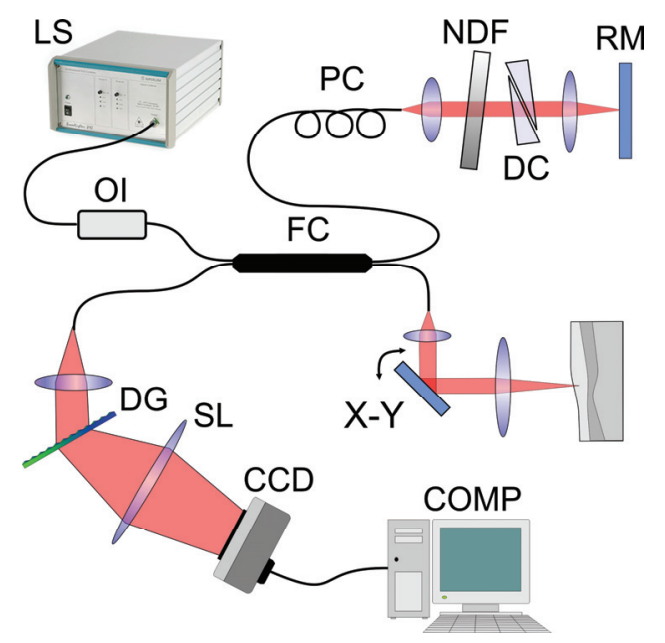

Fig. 2. OCT tomograph used in this study: LS - light source, OI - optical isolator, FC - fibre coupler, PC - polarisation controller, NDF - neutral density filter, DC - dispersion compensator, RM - reference arm mirror, XY - scanner, $\mathrm{DG}$ - diffraction grating, $\mathrm{SL}$ - spectrograph lens, CCD - linear CCD camera P. Targowski et al. "Picosecond laser ablation system with process control by Optical Coherence Tomography", Proc of SPIE
7391, p. 7391-15

Copyright 2009 Society of Photo-Optical Instrumentation Engineers. One print or electronic copy may be made for personal use only. Systematic reproduction and distribution, duplication of any material in this paper for a fee or for commercial purposes, or modification of the content of the paper are prohibited. 
In the OCT system utilised in this study the spectral modality of detection is adopted as described in detail previously[5]. Briefly: information on the locations of back-scattering and reflecting centres distributed within the object along the scanning beam is encoded into the interference signal by means of spectral fringes superimposed on light source spectrum. Therefore, to extract this information the SOCT tomograph is equipped with a spectrograph comprising fast CCD camera as a detector. The custom design spectrometer is build of a volume phase holographic grating (DG) with 1200 grooves/mm and an achromatic lens (SL) which focuses the spectrum on a 12 bit single line CCD camera (2048 pixels, 12 bit A/D conversion). The spectral fringe pattern registered by this detector is then transferred to the personal computer. This signal after the Fourier transformation yields one line of the cross-sectional image (A-scan). Scanning across the sample with transversal scanner (X-Y) enables collecting a 2-D cross-sectional image (B-scan). Additional scanning in the perpendicular direction gives 3-D information about the spatial structure of the sample. Due to the high speed of data collection, the tomograph is capable of 3D (volume) data collection within seconds.

\subsection{Samples}

Especially prepared samples of various varnish layers on oil paintings and cardboard support were used for the experiments. Samples at least two years old have been chosen to make certain that the drying process was fully completed.

Table 1. Varnishes used in this study

\begin{tabular}{|cl|}
\hline Varnish No & \multicolumn{1}{c|}{ Description } \\
\hline 1 & $\begin{array}{l}\text { commercial dammar varnish from Maimeri, applied in 5 layers, } \\
\text { thickness = approx. } 300 \mu \mathrm{m},\end{array}$ \\
2 & $\begin{array}{l}\text { commercial acrylic varnish from Royal Talens, no 115, applied in 4 layers, } \\
\text { thickness }=100 \div 150 \mu \mathrm{m},\end{array}$ \\
3 & $\begin{array}{l}\text { home-made varnish intended to mimic old recipes containing 1 part of } \\
\text { linseed oil, 1 part of dammar and 1 part of turpentine oil, applied in } 5 \\
\text { layers, thickness }=400 \div 500 \mu \mathrm{m} .\end{array}$ \\
\hline
\end{tabular}

\section{RESULTS}

The experiment was conducted as follows: after ablation of thin layer of varnish from the rectangular area $(30 \times 5 \mathrm{~mm})$ of the sample, the OCT volume scan (Fig. 3) was performed in this place. Then the procedure was repeated until desired varnish layer was removed. In the figure an exemplary tomogram is shown. Data is composed of $150 \mathrm{~B}$-scans taken in plane perpendicular to the ablation grooves. In addition to the internal structure of the sample seen as a volume image, the air - varnish interface is shown in colour. This surface data has been recovered from the OCT scattered data and presented here for better visibility with elevation coded in colour intensity. See out other contribution in this volume [13] for details. This high resolution profilometric information may be further processed to obtain more comprehensive data. As it can be seen in figure ablation depth gradually increases from right to left. Since the scanning speed remained constant during whole experiment it suggests a slow increase of pulse energy during laser scanning. It was most likely caused by warm-up of the laser from the start of operation to attain nominal laser beam parameters. Another reason of gradual increase of output energy of the laser could be caused by warm-up of two-stage frequency converter to nominal temperature of phase matching. As a result, laser fluency increased. This in turn gave rise to the gradual increase of the ablation depth. 


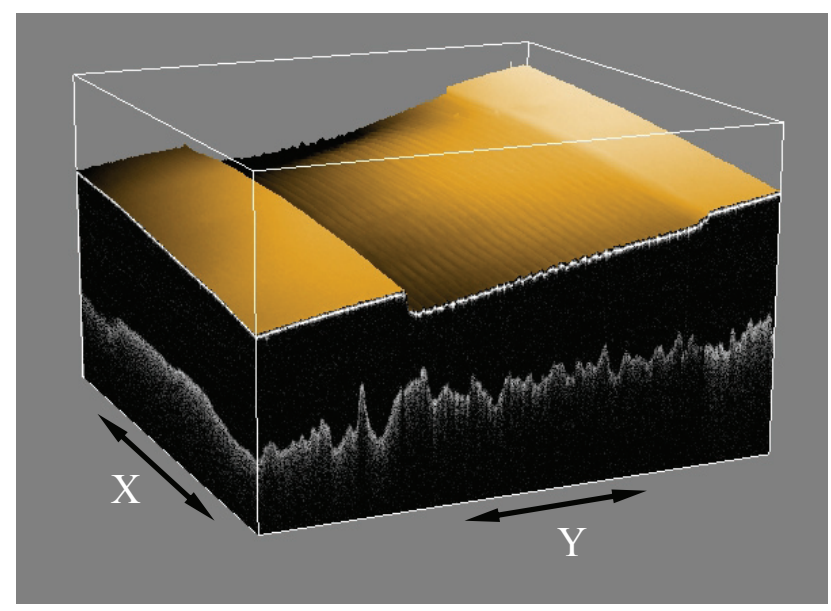

Fig. 3. 3D OCT tomogram from the sample of varnish No 3 (Table 1) after 10 scans with $0.5 \mathrm{~mJ}$ laser pulses of spot diameter $=100 \mu \mathrm{m} . \mathrm{X}$ and $\mathrm{Y}$ indicate directions of fast and slow movements of the sample during ablation. OCT image is not corrected for refraction and extended vertically: cage dimensions are $9.5 \times 9.5 \times 1.5 \mathrm{~mm}$. Tomograms are shown in false colours: light spots indicate high scattering

To address this effect quantitatively, the maps of ablation rates were generated from the OCT data. In the first step the surface elevation maps were compared with the surface map taken before the ablation process. This way the ablation depth may be determined. It is worthwhile to note, that due to the high precision of the elevation maps and to the fact, that the sample was moved during ablation between collecting OCT data, it was necessary to numerically adjust profile maps by correlation of non ablated areas (left- and right-hand side in Fig. 3). Exemplary result is presented in Fig. 4. In the figure ablation depth or the thickness of material removed is shown for the sample from Fig. 3.

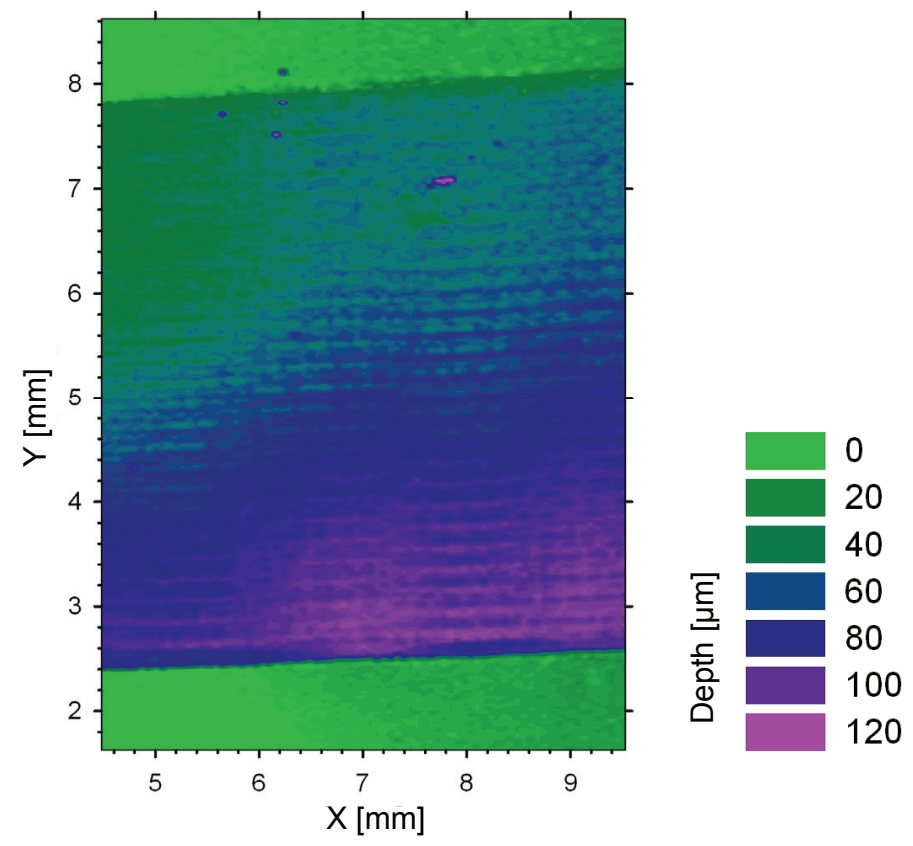

Fig. 4. Example of the map of ablation depth after 10 laser scans over varnish No 3 (Table 1), obtained from data shown in figure 2. The thickness of material removed is colour coded. Green strips in upper and lower part of image denote areas not treated 7391, p. $7391-15$

Copyright 2009 Society of Photo-Optical Instrumentation Engineers. One print or electronic copy may be made for personal use only. Systematic reproduction and distribution, duplication of any material in this paper for a fee or for commercial purposes, or modification of the content of the paper are prohibited. 
In the second step data obtained during all ablation process are analysed together. At each point of the surface the ablation rate is determined (Fig. 5). To do so, depths achieved after consecutive ablation scans were considered as a function of the scan number. The slopes of this dependence were calculated and regarded to be an ablation rates.
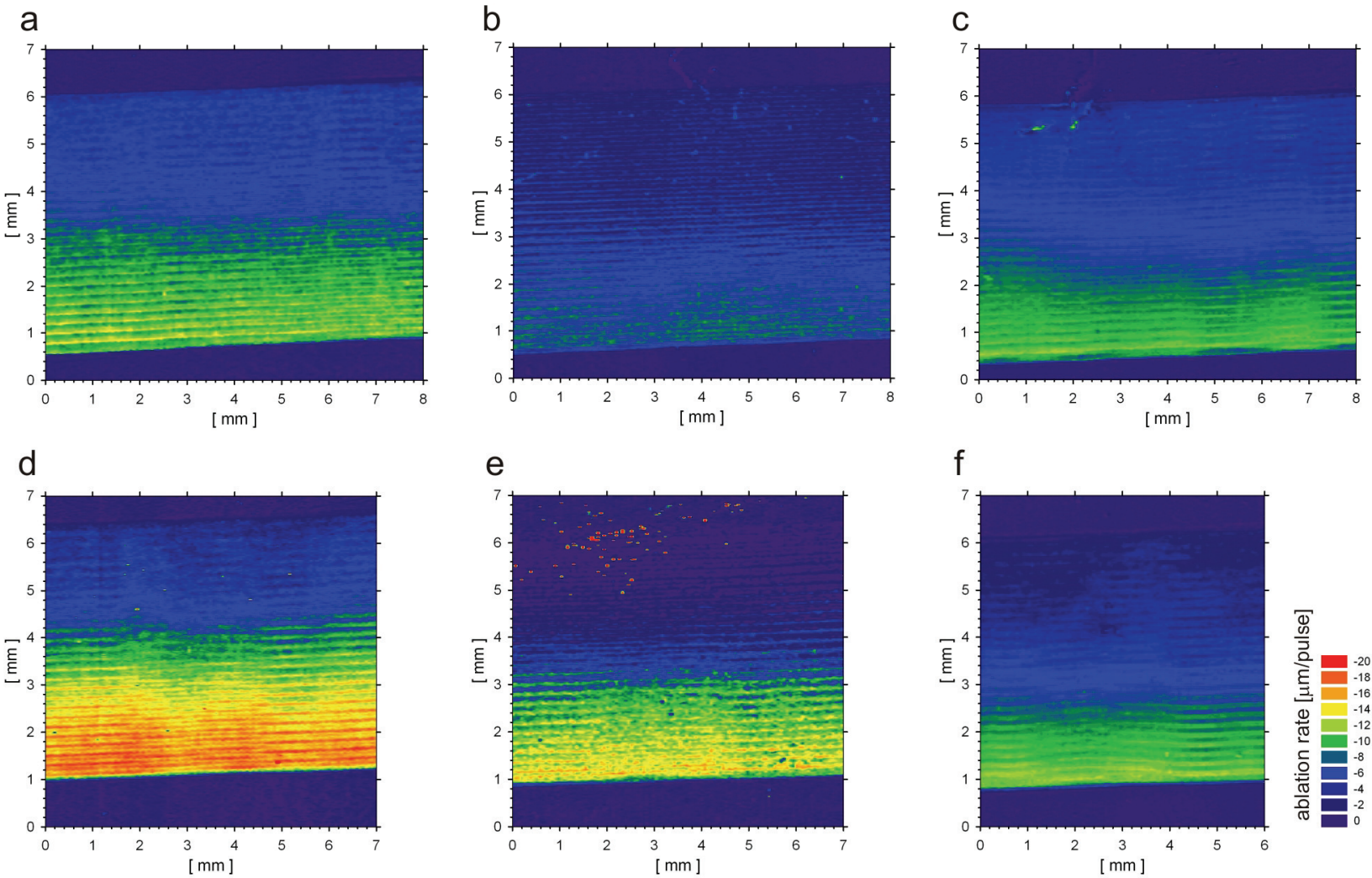

Fig. 5. Surface distributions of ablation rates (shown all in the same colour scale) for various varnishes and spot sizes; a - c: spot size $=100 \mu \mathrm{m}, \mathrm{d}-\mathrm{f}$ : spot size $=130 \mu \mathrm{m}$; a,d: varnish No 1, b,e: varnish No 2; c, f: varnish No $3-$ see Table 1 for details

\section{CONCLUSIONS}

Performed experiment has shown that ultraviolet laser radiation combined with short pulse durations is in principle well suited for varnish removal. The applied laser, emitting pulses of $70 \mathrm{ps}$ in duration at wavelength of $266 \mathrm{~nm}$, allowed to efficient and precise removal of varnish layers, without any clear side effects. Two factors are decisive for this observation: commonly known increase of absorption coefficient for UV rage of wavelength radiation and a short duration of laser pulses. The former limits the absorption of laser energy to a very thin surface layer of the sample, the latter limits the heat dissipation within the sample. The heat dissipation is controlled by the thermal relaxation time of the material and desirable effect of energy concentration in a very thin, sub-micron surface layer is achieved when this time is longer than the pulse duration. This is a case for varnishes which due to being organic materials usually have thermal relaxation times as long as several nanoseconds [14].

It should be noted that varnish removal was performed at low pulse energies, only a little bit above the ablation threshold. As a result the every single pulse removed very small amount of the sample material. However, the high repetition rate and the proper scanning speed gave the sufficient overlap factor for efficient and precise varnish removal, free from any side effects like discolorations, cracks or exfoliations. Together with process control by the OCT this allowed to terminate the ablation procedure and leave a few micrometer of the varnish layer on top of the painted substrate, is desired. The purpose of this layer is to act as a filter protecting the underlying pigments from further exposure.

P. Targowski et al. "Picosecond laser ablation system with process control by Optical Coherence Tomography", Proc of SPIE 7391, p. $7391-15$

Copyright 2009 Society of Photo-Optical Instrumentation Engineers. One print or electronic copy may be made for personal use only. Systematic reproduction and distribution, duplication of any material in this paper for a fee or for commercial purposes, or modification of the content of the paper are prohibited. 
Despite a number of advantages, during the removal of old varnish layers with picosecond UV lasers, a special attention should be paid. The pulse energy as well as the laser spot size on the target has to be selected carefully to minimize possible side effects. Therefore, before implementation of laser cleaning procedure, some tests should be made to evaluate the proper and optimal parameters of laser radiation such as laser fluency and overlap factor. To conduct these tests conveniently and to continue with the ablation process safely, the OCT monitoring is well suited. It allows not only, as it has been shown previously, to qualitatively monitor the ablation process, but also to quantitatively determine the ablation rates, separately for every point of the surface treated.

\section{ACKNOWLEDGEMENT}

This work was supported by Polish Government Research Grants through the years 2008 -2011. MS and EK gratefully acknowledge additional support from European Social Fund and Polish Government within Integrated Regional Development Operational Programme, Action 2.6, by project "Stypendia dla doktorantów 2008/2009 - ZPORR" of Kuyavian-Pomeranian Voivodeship.

\section{REFERENCES}

[1] Fotakis C., Anglos D., Zafiropulos V., Georgiou S., Tornari V., [Lasers in the Preservation of Cultural Heritage, Principles and Applications] CRC Press, Boca Raton, 229-258 (2006).

[2] Góra, M., Targowski, P., Rycyk, A. and Marczak, J. "Varnish ablation control by Optical Coherence Tomography" Laser Chemistry 2006, 1-7 (2006) DOI: DOI:10.1155/2006/10647.

[3] Góra, M., Rycyk, A., Marczak, J., Targowski, P. and Kowalczyk, A., "From medical to art diagnostics OCT: a novel tool for varnish ablation control", Proceedings of SPIE 6429, 64292V (2007).

[4] Góra, M., Targowski, P., Kowalczyk, A., Marczak, J. and Rycyk, A., "Fast spectral optical coherence tomography for monitoring of varnish ablation process", Lacona 7 - International conference on Lasers in the Conservation of Artworks, Madrid, Spain, September 17 -21 2007, Taylor \& Francis Group, 23-26 (2008).

[5] Targowski, P., Rouba, B., Góra, M., Tymińska-Widmer, L., Marczak, J. and Kowalczyk, A., "Optical coherence tomography in art diagnostic and restauration", Applied Physics A: Materials Science and Processing 92, 1-9 (2008).

[6] Targowski, P., Góra, M., Marczak, J., Rycyk, A. and Rouba, B. J. "Optical coherence tomography holds promise for conserving art" newsroom.spie.org, (2009) DOI: 10.1117/2.1200904.1589.

[7] Liang, H., Cid, M., Cucu, R., Dobre, G., Podoleanu, A., Pedro, J. and Saunders, D., "En-face optical coherence tomography-a novel application of non-invasive imaging to art conservation". Optics Express 13(16), 6133-6144 (2005).

[8] Targowski, P., Rouba, B., Wojtkowski, M. and Kowalczyk, A., "The application of optical coherence tomography to non-destructive examination of museum objects", Studies in Conservation 49(2), 107-114 (2004).

[9] Yang, M. L., Lu, C. W., Hsu, I. J. and Yang, C. C., "The use of Optical Coherence Tomography for monitoring the subsurface morphologies of archaic jades", Archaeometry 46, 171-182 (2004).

[10] "Complete list of papers published on application of OCT to artwork" - retrieved 20 February 2009, from www.oct4art.eu.

[11] Marczak, J., Ostrowski, R., Rycyk, A., Strzelec, M., Szmelter, I. and Koss, A., "Investigations of varnish removal using modified Er:YAG laser", Lasers in the Conservation of Artworks, LACONA VI Proceedings, Vienna, Austria, Sept. 21 - 25, 2005, 71 (2007).

[12] Marczak, J., Strzelec, M., Ostrowski, R., Rycyk, A., Sarzyński, A., Skrzeczanowski, W., Koss, A., Szambelan, R., Salimbeni, R., Siano, S., Kolar, J., Strlič, M., Márton, Z., Sánta, I., Kisapáti, I., Gugolya, Z., Kántor, Z., Barcikowski, S., Engel, P., Pires, M., Guedes, J., Hipólito, A., Santos, S., Dement'ev, A. S., Švedas, V., Murauskas, E., Slavinskis, N., Jasiunas, K. and Trtica, M., "Advanced laser renovation of old paintings, paper, parchment and metal objects", Lacona 7 - International conference on Lasers in the Conservation of Artworks, Madrid, Spain, September 17 -21 2007, Taylor \& Francis Group, 263-270 (2007).

P. Targowski et al. "Picosecond laser ablation system with process control by Optical Coherence Tomography", Proc of SPIE 7391, p. $7391-15$

Copyright 2009 Society of Photo-Optical Instrumentation Engineers. One print or electronic copy may be made for personal use only. Systematic reproduction and distribution, duplication of any material in this paper for a fee or for commercial purposes, or modification of the content of the paper are prohibited. 
[13] Sylwestrzak, M., Kwiatkowska, E. A., Karaszkiewicz, P., Iwanicka, M. and Targowski, P., "Application of graphically oriented programming to imaging of structure deterioration of historic glass by Optical Coherence Tomography ", Proc SPIE 7391, 739109-1 (2009).

[14] Gamalay, E. G., Rode, A. V., Luther-Davies, B. and Tikhonchuk, V. T., "Ablation of solids by femtosecond lasers: Ablation mechanism and ablation thresholds for metals and dielectrics", Phys. Plasmas 9(3), 949-957 (2002).

P. Targowski et al. "Picosecond laser ablation system with process control by Optical Coherence Tomography", Proc of SPIE 7391, p. 7391-15

Copyright 2009 Society of Photo-Optical Instrumentation Engineers. One print or electronic copy may be made for personal use only. Systematic reproduction and distribution, duplication of any material in this paper for a fee or for commercial purposes, or modification of the content of the paper are prohibited. 